\section{Depression as the First \\ Clinical Manifestation of Antiphospholipid Syndrome: A Case Study}

\author{
Ali Ercan Altınöz ${ }^{1 *}$, Şengül Tosun Altınöz ${ }^{2}$ and Behçet Coşar ${ }^{2}$ \\ ${ }^{1}$ Ankara Penal Institution Campus State Hospital, Psychiatry, Ankara, \\ Turkey \\ ${ }^{2}$ Department of Psychiatry, Faculty of Medicine, Gazi University, Ankara, \\ Turkey \\ ${ }^{3}$ Department of Psychiatry, Faculty of Medicine, Gazi University, Ankara, \\ Turkey
}

\begin{abstract}
Objective: Antiphospholipid Syndrome (APS) is an autoimmune disorder that usually manifests as arterial or venous thromboembolic events and recurrent spontaneous abortions. As the disease affects many organs by obstructing the small vessels, it can present with a variety of symptoms. Neuropsychiatric symptoms are rarely the first to be observed. This report describes the case of a woman with no known disease who presented at a psychiatric outpatient clinic with aversion and cognitive impairment and was diagnosed with APS.

Conclusion: It is important to bear autoimmune diseases in mind when evaluating young adults with complicated symptoms at psychiatric outpatient clinics.
\end{abstract}

Keywords: Antiphospholipid syndrome; Cognitive impairment; Depression

\section{Introduction}

Antiphospholipid Syndrome (APS) is an autoimmune disease that manifests as arterial or venous thromboembolic events and recurrent spontaneous abortions [1]. It is diagnosed by demonstrating the presence of antibodies, according to the Sapporo criteria updated in 2010 [2]. Primary APS is the presence of antiphospholipid antibody relating to a vaso-occlusive event without an underlying disease, and secondary APS has an underlying disease such as malignancy or Systemic Lupus Erythematosus (SLE) [3]. Antiphospholipid antibody is present in $20-40 \%$ of SLE patients, compared with less than $5 \%$ in healthy populations [2]. In the general population, the frequency of APS is not clearly known [2]. It can present with various clinical symptoms because it can affect many organs by obstructing small

*Corresponding author: Ali Ercan Altınöz, M.D., İşçiBloklari Mh., Muhsin Yazicioğlu, Cd. No: 44, KelebekSitesi D: 28, PK:06530, Çankaya / Ankara / Turkey, Tel: 00903122540270; E-mail: ercanaltinoz@hotmail.com

Citation: Altınöz AE, Altınöz ST, Coşar B (2015) Depression as the First Clinical Manifestation of Antiphospholipid Syndrome: A Case Study. J Clin Stud Med Case Rep 2: 004.

Received: September 19, 2014; Accepted: December 30, 2014; Published: January 13, 2015 vessels. In cases of central nervous system involvement, APS presents with a variety of neuropsychiatric symptoms [1,4,5]. Although these symptoms frequently appear in the late phase of the disease, sometimes they manifest first [6]. It is important to begin treatment as soon as the disease is recognized [6].

In this report, we describe the case of a woman with no known history of any disease who presented to a psychiatric outpatient clinic with symptoms of aversion and cognitive impairment and was diagnosed with APS.

\section{Case}

A 29-year-old woman, married with no children, presented to a psychiatric outpatient clinic with symptoms of aversion, anhedonia, and amnesia. Following the death of her sibling in a car accident 4-5 months previously, she was getting easily tired, was not enjoying her life, had lost interest, and, especially in the past month, was forgetting names and words and had difficulty in understanding sentences. No diagnosis had been made nor any treatment given because she had no known systemic disease.

During psychiatric evaluation, it was observed that she appeared older than her chronological age, her self-care was insufficient, and she had significant psychomotor retardation. She had memory impairment, but perception was not distorted. Her thought content was poor, and anhedonia was present. She was conscious and oriented. She did not have suicidal ideation, her mood was depressed, and her affect was blunted. She did not spontaneously start conversation but only answered questions; however, her conversation was goal directed.

The Hospital Anxiety and Depression Score (HADS) was 8 . Routine biochemistry tests were requested, including complete blood count, thyroid functioning tests, and vitamin B12 and folic acid levels. Following the diagnosis of depression and mild cognitive impairment, escitalopram was prescribed ( $5 \mathrm{mg} /$ day for the first week and $10 \mathrm{mg} /$ day there after).

After 2 weeks, the patient's test results were received and she came to the follow-up appointment. She mentioned that she had twice lost her way home-where she has been living for the past 10 years-and that such amnesia problems had been frequent in recent days. She also indicated that her symptoms were worsening.

The complete blood count test revealed no abnormalities except thrombocytopenia $(72000 / \mathrm{mL})$. The standardized Mini Mental State Examination (MMST) revealed a score of 25. When her past history was taken in detail, we found that she had become pregnant three times: all three had miscarried in the first trimester. MR imaging was requested, and she was referred to the rheumatology clinic with a provisional diagnosis of early APS. Escitalopram was stopped.

Rheumatological examination found anticardiolip in IgG and IgM antibodies, and the diagnosis of APS was confirmed. Steroid treatment was begun, and after a week, acetylsalicylic acid (100 mg/ day) was added. MRI imaging detected hyperintense nonspecific multiple glotticfocuses at $\mathrm{T} 2$. 
At the 3-month follow-up, the patient was still taking acetylsalicylic acid ( $100 \mathrm{mg} /$ day); her previous complaints had almost disappeared, she feltless exhausted, and she was no longer preoccupied. MMST and HADS scores were 30 and 2, respectively.

\section{Discussion}

APS is rare in children and elderly but frequently seen in young adults [7]. In more than one-fifth of patients with APS, the first symptoms are venous thromboembolism, thrombocytopenia, recurrent abortions, or lived or eticularis [2]. Our patient was diagnosed at 29 years of age. It was discovered that, although she had a history of recurrent abortions, APS had not been considered. It is rare situation for the first symptom of APS to be neuropsychiatric [2], but in our patient, because the recurrent abortions had not led to diagnosis, cognitive impairment was the indicator. Central nervous system involvement is frequent in APS, presenting with symptoms such as delirium, seizure, transient ischemic attack, stroke, psychosis, sensory neural hearing loss, and mood disorders [8]. Cognitive impairment is another of these symptoms. Between $11 \%$ and $42 \%$ of patients with APS have cognitive impairment [9-11]. Although this impairment is associated with areas of verbal fluency and complicated attention, neuropsychological tests were not performed in our patient, so the areas influenced could not be determined [11]. It has been previously reported that the level of cognitive impairment is associated with anticardiolipin antibody level and white matter hyperintensity on MRI imaging [11].

The treatment of APS is controversial. Some reports indicate that steroid treatment is ineffective in terms of course of the disease, while others recommend its inclusion $[2,12,13]$. However, the necessity of antiplatelet and anticoagulant treatments to prevent secondary thrombus is generally agreed upon, and acetylsalicylic acid and low molecular weight heparin are the recommended agents $[2,13,14]$. In our patient, acetylsalicylic acid $(100 \mathrm{mg} /$ day $)$ was administered along with mini-pulse steroid treatment; after a while, the dose of the steroid treatment was lowered and then stopped. Some cases of APS can have a dramatic response to treatment; nevertheless, it is possible to reverse the course of autoimmune diseases with central nervous system involvement by early diagnosis and early treatment $[6,13]$. In our patient, the diagnosis was made within a month of central nervous system involvement, and then the treatment was begun. After treatment, the symptoms of preoccupation, aversion, amnesia, and anhedonia disappeared.

As diagnostic and therapeutic delay may lead to a more extensive spread of the autoimmune aggression and damage to the central nervous system it is important for clinicians to recognize and treat APS effectively [15]. Furthermore brain imaging examinations were mentioned to prevent a delay in the detection of a structural lesion and facilitate the early intervention with good prognosis [16].

In summary, APS is an autoimmune disease concomitant with neurological symptoms. Although its treatment is debated and may vary from patient to patient, early diagnosis and appropriate treatment could change the course of the disease. It is important to keep autoimmune diseases in mind when evaluating young adults applying to an outpatient psychiatry clinic with complicated symptoms.

\section{References}

1. Hughes GR (1993) The antiphospholipid syndrome: ten years on. Lancet 342: 341-344.

2. Ruiz-Irastorza G, Crowther M, Branch W, Khamashta MA (2010) Antiphospholipid syndro Lancet 376: 1498-1509.

3. Amigo MC, Khamashta MA (2000) Antiphospholipid (Hughes) syndrome in systemic lupus erythematosus. Rheum Dis Clin North Am 26: 331-348.

4. Arnson Y, Shoenfeld Y, Alon E, Amital H (2010) The antiphospholipid syndrome as a neurological disease. Semin Arthritis Rheum 40: 97-108.

5. Etemadifar M, Dehghani L, Tahani S, Toghianifar N, Rahaimi M, et al. (2013) Neurological manifestations in patients with antiphospholipid syndrome. Iran J Neurol 12: 172-175.

6. Lai JY, Wu PC, Chen HC, Lee MB (2012) Early neuropsychiatric involvement in antiphospholipid syndrome. Gen Hosp Psychiatry 34: 579.

7. Hoffman R (2005) Hematology: Basic Principles And Practice. Elsevier Churchill Livingstone 2151-2160.

8. Sanna G, Bertolaccini ML, Mathieu A (2003) Central nervous system lupus: a clinical approach to therapy. Lupus 12: 935-942.

9. Fetoni V, Grisoli M, Salmaggi A, Carriero R, Girotti F (2000) Clinical and neuroradiological aspects of Sneddon's syndrome and primary antiphospholipid antibody syndrome. A follow-up study. Neurol sci 21: 157-164.

10. Tourbah A, Piette JC, Iba-Zizen MT, Lyon-Caen O, Godeau P, et al. (1997) The natural course of cerebral lesions in Sneddon syndrome. Arch Neurol 54: 53-60.

11. Tektonidou MG, Varsou N, Kotoulas G, Antoniou A, Moutsopoulos HM (2006) Cognitive deficits in patients with antiphospholipid syndrome: association with clinical, laboratory, and brain magnetic resonance imaging findings. Arch Intern Med 166: 2278-2284.

12. Lassere M, Empson M (2004) Treatment of antiphospholipid syndrome in pregnancy--a systematic review of randomized therapeutic trials. Thromb Res 114: 419-426.

13. Cuadrado MJ (2002) Treatment and monitoring of patients with antiphospholipid antibodies and thrombotic history (Hughes syndrome). Curr Rheumatol Rep 4: 392-398.

14. George D, Erkan D (2009) Antiphospholipid syndrome. Prog Cardiovasc Dis 52: $115-125$

15. Carecchio M, Cantello R, Comi C (2014) Revisiting the molecular mechanism of neurological manifestations in antiphospholipid syndrome: beyond vascular damage. J Immunol Res 2014: 239398.

16. Li CH, Chou MC, Liu CK, Lai CL (2013) Antiphospholipid syndrome presenting as progressive neuropsychiatric disorders: two case reports. Neuropsychiatr Dis Treat 9: 739-742. 